\title{
The Mechanism of Allosteric Coupling in Choline Kinase $\alpha 1$ Revealed by the Action of a Rationally Designed Inhibitor**
}

\author{
María Sahún-Roncero, Belén Rubio-Ruiz, Giorgio Saladino, Ana Conejo-García, \\ Antonio Espinosa, Adrián Velázquez-Campoy, Francesco Luigi Gervasio, Antonio Entrena, and \\ Ramon Hurtado-Guerrero*
}

Choline kinase $(\mathrm{CHOK})$ is a homodimeric enzyme that catalyzes the transfer of a phosphate group from ATP to choline to produce ADP and phosphocholine (PCho; Figure $1 \mathrm{~A}$ ), the first step in the Kennedy pathway leading to the synthesis of phosphatidylcholine (PtdCho).$^{[1]}$ An overexpression of the $\alpha$ isoform of $\mathrm{CHOK}$ has been associated with human cancers because PtdCho is required for the growth of cancer cells. ${ }^{[2]}$ These findings have motivated the development of $\mathrm{CHOK} \alpha$ inhibitors, such as hemicholinium-3 (HC-3; Figure $1 \mathrm{~B}),{ }^{[3]}$ and a large number of HC-3 derivatives. ${ }^{[4,5]}$ Although early studies reported evidence of cooperative binding in $\mathrm{CHOK},{ }^{[6]}$ the binding of $\mathrm{HC}-3$ does not involve allosteric coupling between the two monomers. We recently synthesized a new family of monocationic $\mathrm{CHOK}$ inhibitors that, in contrast to $\mathrm{HC}-3$, are non-symmetrical. ${ }^{[7]}$ Herein, we characterized one of these compounds (compound 1; Figure $1 \mathrm{~B}$ and Supporting Information, Table S1) and found evidence of a significant allosteric coupling between the two monomers characterized by negative cooperativity and a broken symmetry.

Allostery is the process whereby the binding of a ligand to a biological macromolecule (protein) induces an effect on a distant functional site. Two classical models have been proposed to describe allostery: the Monod-Wyman-Changeaux (MWC) or concerted model ${ }^{[8]}$ and the sequential model ${ }^{[9]}$ The first posits that the conformational changes in the different monomers of the bio-macromolecule are strongly coupled and that symmetry is conserved among the mono- mers. The second assumes a strong coupling between binding and conformational changes. More recently molecular dynamics simulations helped in linking large-amplitude motions to allosteric mechanisms ${ }^{[10]}$ and an ensemble view of allostery has been proposed, reconciling multiple protein conformations with allosteric signaling. ${ }^{[11]}$ Herein, by using a combination of experimental (isothermal titration calorimetry (ITC), X-ray crystallography) and computational approaches, we observed a broken inter-monomer symmetry leading to negative cooperativity and a dynamic recovery of the symmetry owing to a fast inter-conversion of conformers.

Compound 1 (Figure 1B) has a 4-substituted pyridinium and an adenine moiety connected by a flexible linker ${ }^{[7]}$ Its adenine moiety docks to the ATP site of the enzyme, while the pyridinium fragment occupies the choline binding site. In stark contrast to HC-3, 1 induces negative cooperativity. It exhibits the expected biphasic titration curves and a cooperativity constant $\alpha$ of 0.2 , corresponding to a cooperativity $\Delta G$ of $0.95 \mathrm{kcal} \mathrm{mol}^{-1}$ and a Hill coefficient of 0.62 (Figure $1 \mathrm{C}$, Table S1 and Supporting Information). Compound 1 first binds to one monomer of the dimeric CHOK $\alpha 1$ with a dissociation constant $K_{\mathrm{d} 1}$ of $38 \mathrm{~nm}$. The binding to one monomer induces negative cooperativity on the second monomer, leading to a higher $K_{\mathrm{d} 2}$ of $190 \mathrm{~nm}$ (Table S1). An allosteric coupling between the two monomers has been reported in S. cerevisiae CHOK ( $\mathrm{ScCHOK}$ ) where positive cooperativity upon ATP binding was observed. ${ }^{[6 b]}$ We also checked in CHOK $\alpha 1$ whether AMP-PNP, an analogue of
[*] M. Sahún-Roncero, Dr. A. Velázquez-Campoy,

Dr. R. Hurtado-Guerrero

Institute of Biocomputation and Physics of Complex Systems (BIFI), University of Zaragoza,

BIFI-IQFR (CSIC) Joint Unit, Mariano Esquillor s/n, Campus Rio

Ebro, Edificio I+D; Fundacion ARAID

Edificio Pignatelli 36 (Spain)

E-mail: rhurtado@bifi.es

Dr. B. Rubio-Ruiz, Dr. A. Conejo-García, Prof. A. Espinosa,

Prof. A. Entrena ${ }^{[+]}$

Departamento de Química Farmaceútica y Orgánica, Facultad de Farmacia, Universidad de Granada

c/Campus de Cartuja s/n, Granada (Spain)

Dr. G. Saladino, Dr. F. L. Gervasio ${ }^{[+]}$

Computational Biophysics group,

Spanish National Cancer Research Center

Melchor Fernández Almagro, 3, Madrid (Spain)

and

Department of Chemistry, University College London

20 Gordon Street, London WC1H OAJ (UK) $\left.{ }^{+}\right]$These authors contributed equally.

[**; We thank the European Synchrotron Radiation Facility (ESRF) at Grenoble and the Diamond Light Source (DLS) at Oxford, specifically beamlines ID23-1 and 103, respectively. We thank the Fundación Agencia Aragonesa para la Investigación y el Desarrollo (ARAID, Spain), the Ministerio de Economía y Competitividad (MEC, Spain, grants BFU2010-19504, BFU2010-19451, SAF200911955, and BIO2010-20166), the Consejería de Innovación, Ciencia y Empresa of the Junta de Andalucía (grant P07-CTS-03210), the Ministerio de Educación for a FPU fellowship (AP2007-03115) to B.R.-R., and Diputación General de Aragón (Grupo Protein Targets, B89) and the Comunidad Autónoma de Madrid S2010-BMD-2457 (BIPEDD2). This work was granted access to the HPC resources of HECToR, within the Distributed European Computing Initiative (FP7/2007-2013, RI-283493).

Supporting information for this article (experimental details) is available on the WWW under http://dx.doi.org/10.1002/anie. 201209660. 
A<smiles>Nc1ncnc2c1ncn2C(O)C(O)COP(=O)([O-])OP(=O)([O-])OP(=O)([O-])[O-]</smiles>
ATP

B<smiles>C[N+](C)(C)CCO</smiles>

HC-3<smiles>Nc1ncnc2c1ncn2C(O)C(O)COP(=O)(O)OP(=O)([O-])[O-]</smiles><smiles>CN(C)CCOP(=O)([O-])OCCO</smiles><smiles>CN(C)c1cc[n+](Cc2ccc(Cc3ccc(Cn4cnc5c(N)ncnc54)cc3)cc2)cc1</smiles>

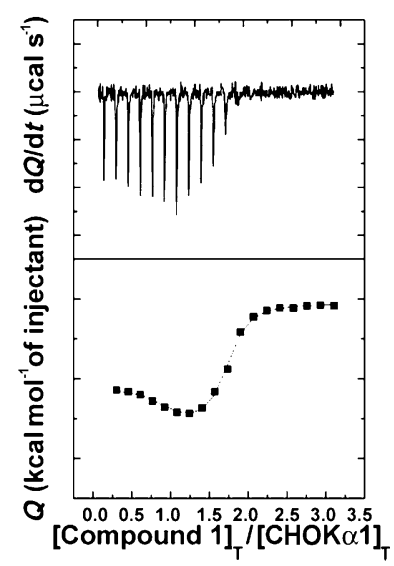

Figure 1. Biophysical characterization of compound 1. A) Scheme of the CHOK enzymatic reaction. B) Chemical formulas of 1 and HC-3. C) ITC data for the binding of 1 to CHOKal. Top: Raw thermogram (thermal power versus time). Bottom: Binding isotherm (normalized heats versus molar ratio). See Table S1 for the thermodynamic and $K_{d}$ values for 1 and HC-3.

ATP, shows cooperativity effects, but we did not find any (Table S1), suggesting that $\mathbf{1}$ induces negative cooperativity by binding in a different manner with respect to ATP.

To test this hypothesis, we solved the crystal structure of CHOK $\alpha 1-1$ at $1.90 \AA$ resolution (Table S2), using molecular replacement using a template (PDB ID: 3G15; see Supporting Information) ${ }^{[3 a]}$ Successive iterative model building and refinement cycles were carried out to produce final models with good refinement statistics (Table S2). The structure is a dimer in which each monomer is formed by a small $\mathrm{N}$ terminal domain that binds ATP and a large C-terminal domain that binds choline (Figure $2 \mathrm{~A}$ ). Whereas the ATP binding site is located in a cleft formed by the $\mathrm{N}$ - and $\mathrm{C}$ - terminal residues, the choline binding site is found in a deep hydrophobic pocket. ${ }^{[12]}$ In agreement with docking predictions, ${ }^{[7]} \mathbf{1}$ fully occupies the $20 \AA$ long active site, from the ATP to the choline binding sites, explaining its potency (Figure $2 \mathrm{~A}$ and Figure $3 \mathrm{~A}, \mathrm{~B}$ ). The main interactions between $\mathbf{1}$ and $\mathrm{CHOK} \alpha 1$ are illustrated in Figure S1.

A comparison of the overall $\mathrm{CHOK} \alpha 1-1$ crystal structure with the apo structure (PDB ID: 2CKO) ${ }^{[12]}$ revealed large conformational changes (Figure $2 \mathrm{~B}$ ). To ensure that crystal packing did not play a role in determining the conformational states, we only used crystal structures sharing the same space group, similar unit cell dimensions, and crystal packing (Table S3). The extent of opening of the binding sites of
A

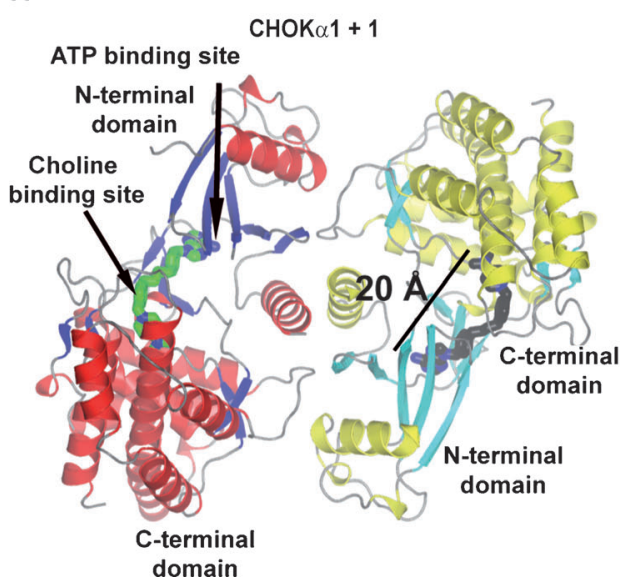

B

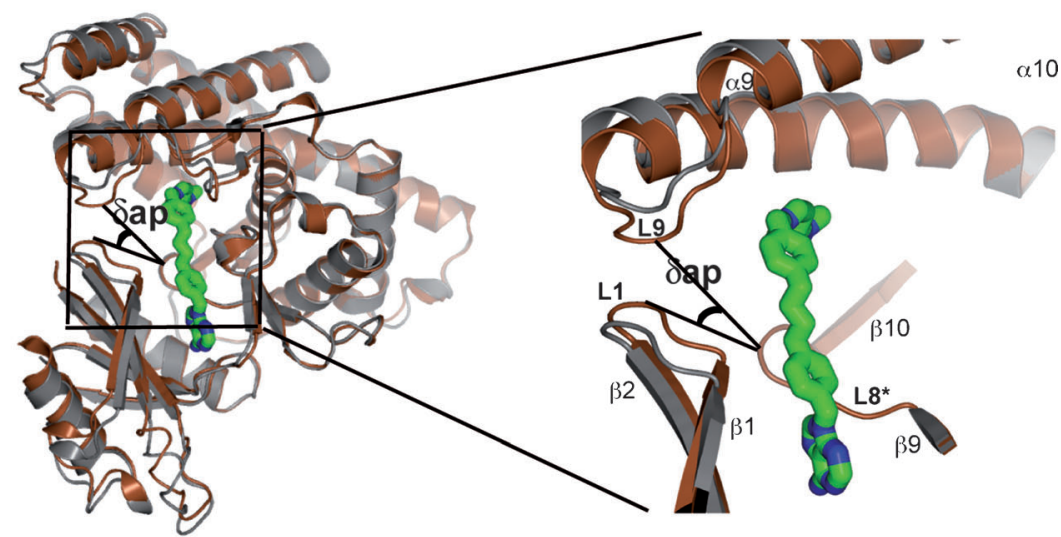

Figure 2. Overall structure of $\mathrm{CHOK \alpha} 1-1$ and conformational states of $\mathrm{CHOK} \alpha$ enzymes. A) Overall crystal structure of $\mathrm{CHOK} \alpha 1$ in complex with 1. Secondary structure elements from the CHOK $\alpha 1$ structure are shown, with $\alpha$-helices in red (monomer A) and yellow (monomer B), and $\beta$ strands respectively in blue and cyan. 1 is shown as a stick model with green and black carbon atoms. B) Illustration of how aperture angle ( $\left.\delta_{\mathrm{ap}}\right)$ is calculated. Monomer B of CHOKa1-1 structure (brown) is superimposed on monomer B of the apo structure (gray). 
A

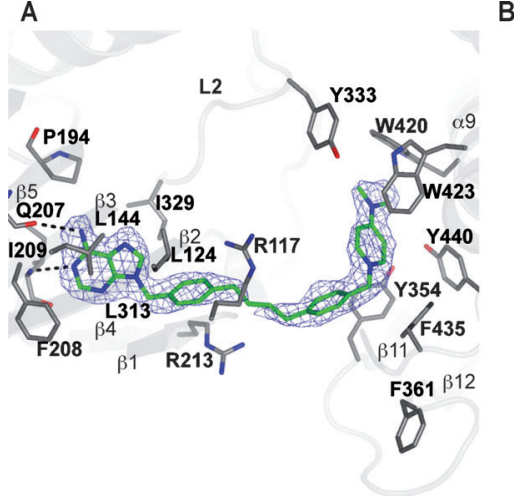

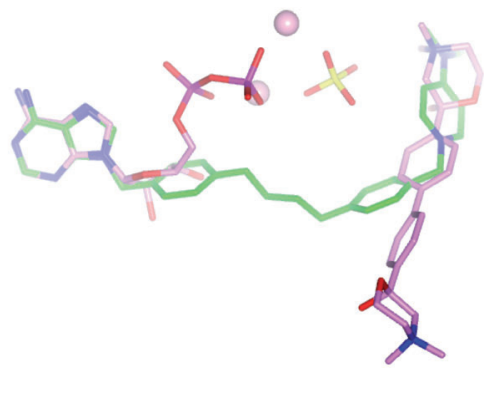

are computationally expensive and the time scales that can be effectively sampled are often insufficient to gather statistically meaningful data on long-range conformational changes. ENMs are based on coarser approximations. The protein is represented by a subset of atoms (often the alpha carbon atom, $\mathrm{C} \alpha$ ) interconnected by a network of elastic springs. Although this model is much simpler with respect to MD, its normal modes are representative of the motions of the protein around its crystallographic structure.

We first performed an analysis based on the perturbation of an anisotropic ENM. ${ }^{[14]}$ The analysis consists of moving the $\mathrm{C} \alpha$ atoms around atom $i$ along each normal mode, giving rise to a new distorted structure. If the structures arising from moving the $\mathrm{C} \alpha$ atoms around atom $j$ along the same eigenvectors are distorted in a correlated way, $i$ and $j$ are allosterically coupled in the structure. The ENM analysis confirms the existence of an allosteric network connecting the two

$\mathrm{CHOK} \alpha$ was assessed by measuring the angle $\delta_{\mathrm{ap}}$ (Figure 2B) defined by the alpha carbon atom of three residues (Ser121 on loop L1, Glu332 on L8*, and Ser431 on L9) in different structures. The loops, L1 and L9 undergo significant conformational changes and the angle they make with L8*, which is relatively rigid, is able to monitor those changes. The value of $\delta_{\text {ap }}$ combined with the root mean square deviations (RMSD) of equivalent alpha carbon atoms from the apo structure (PDB ID: 2CKO) allowed for the identification of three distinct conformational states: open/semi-open, closed, and super-closed states (Figure S2A,B). Open/semi-open states were present in monomers of the apo $\mathrm{CHOK} \alpha 2$, CHOK $\alpha 1-H C-3$ (PDB ID: 3F2R), CHOK $\alpha 1-H C-3-A D P$ (PDB ID: 3G15), and monomer A of CHOKa1-1; a closed state was present in monomer $\mathrm{B}$ of $\mathrm{CHOK} \alpha 1-1$; and a superclosed state was present in CHOK $\alpha 1-\mathrm{PCho}$ (PDB ID: 2CKQ; Figure S2A). The closed and super-closed states followed a trend in which higher RMSD values were associated with a smaller $\delta_{\text {ap. }}$. The crystal structure of the complex with $\mathbf{1}$ is asymmetric. Monomer $\mathrm{B}$ of the $\mathrm{CHOK} \alpha 1-1$ complex differs significantly from monomer A or B of the apo structure, as indicated from an RMSD of $0.9 \AA$ and $1.08 \AA$, respectively (Figure $\mathrm{S} 2 \mathrm{~B}$ ), and it is significantly different from monomer A present in CHOKa1-1 (RMSD of $1.03 \AA$; Figure S2B and Figure S3). Owing to differences in the position of the loops at the cavity entrance, the entrance to the cavity and its size are different (loops L1 and L9, see Figure 2 B), hinting at possible allosteric couplings between the two monomers. This striking asymmetry could provide the structural basis of the negative cooperativity, as proposed by Levitzki et al. ${ }^{[13]}$ (Figure S2, S3).

To further explore the origin of the negative cooperativity and verify the long-range couplings, we performed extensive computational modeling with two complementary methods: all-atom molecular dynamics (MD) simulations and anisotropic elastic network model (ENM) ${ }^{[14]}$ All-atom MD with an explicit solvent can accurately reproduce local and large-scale conformational changes. ${ }^{[15]}$ However, long-MD simulations monomers (Figure S4). Many distant elements, far from the interface, showed a non-trivial allosteric coupling (Figure S4, Figure 4A). The strongest coupling was found between the two $\alpha 7$ helices, which are far from the interface. The $\alpha 7$ helix was also coupled with the two long $\alpha 9$ helices of the other monomer (e.g. $\alpha 9$ of monomer A was coupled with $\alpha 7$ of monomer B and vice versa; Figure S4, Figure 4A). The $\alpha 9$ helices are involved in the opening of the cavity. Finally, the $\alpha 7$ helices in monomers $\mathrm{A}$ and $\mathrm{B}$ are coupled to the interfacial motifs $\alpha 2, \beta 4$, and $\beta 5$, with less intense couplings observed also between $\alpha 9$ and the $\alpha 2 \beta 4 \beta 5$ region (Figure S4, Figure 4A). Thus, there is a coupling between the dimer interface ( $\alpha 2 \beta 4 \beta 5)$ and the choline binding pocket. The allosteric signal flows from the dimer interface to loop L9, involved in the opening/closing mechanism, through helix $\alpha 7$. Because this model involves the perturbation of a specific crystal structure, it is sensitive to the conformation (apo or holo) used for the analysis. Interestingly, some $\alpha 7$ residues are part of the choline binding site and interact with compound $\mathbf{1}$ directly. However, no differences were observed in the choline binding site of the two monomers. Instead, significant differences were observed at the other end of the ligand, the adenine moiety that binds in the kinase ATP binding site. Indeed, in the monomer B, which is more closed and to which $\mathbf{1}$ binds better, the ligand makes stronger (shorter length) hydrogen bonds to Gln207 and Ile209 (Figure 3 A). Based on these observations, it seems unlikely that the binding of the first molecule directly triggers the closure of the L1-L9 loops. Instead, the stronger binding of $\mathbf{1}$ to the ATP binding site, and in particular to the hinge region preceding $\beta 4$ and $\beta 5$, might be responsible for both the L1-L9 loops closure and the perturbation of the other monomer binding site, through the coupling with $\alpha 7$ (Figure 4A).

To verify the coupling between different structural elements in the $\mathrm{CHOK} \alpha 1$ dimer and to investigate the driving force for L1-L9 loop closure and the break of symmetry, we performed fully atomistic MD. The MD simulations in an explicit solvent were performed with 


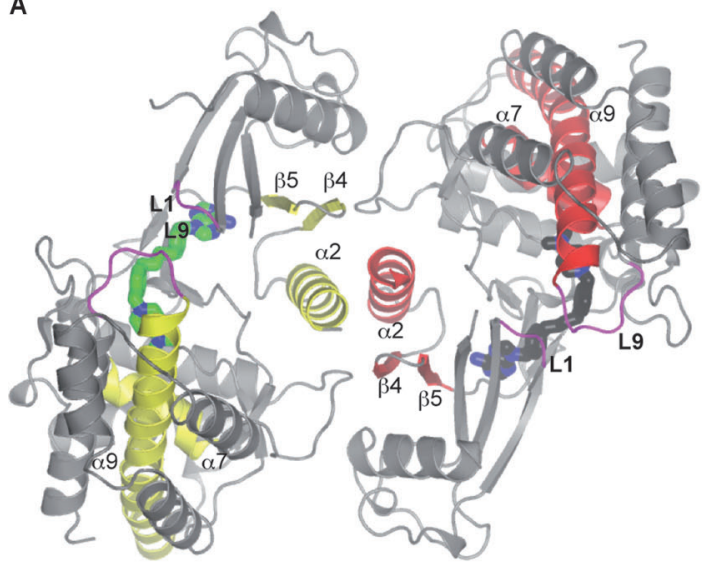

B
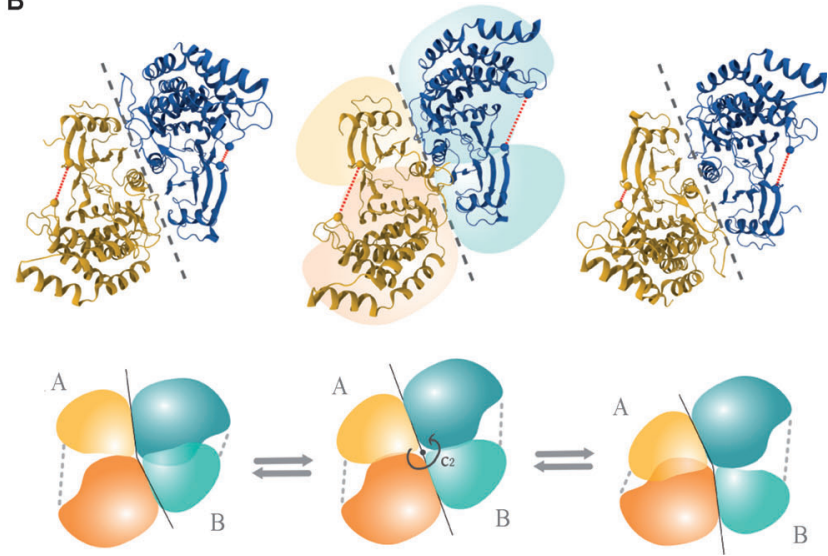

Figure 4. Elastic network analysis and molecular dynamics simulations. A) Overall structure of $\mathrm{CHOK} \alpha 1$ showing the coupled secondary structures and the L1 and L9 loops (see also Figure S4, S5 for the numbering of secondary structures and loops). B) Scheme demonstrating that during the simulation, the protein dimer shifts from a closed conformation of monomer $B$ to a final conformation where monomer $\mathrm{A}$ is closed.

GROMACS 4.5 ${ }^{[16]}$ using the Amber99SB*-ILDN ${ }^{[17]}$ force field for the protein part and $\mathrm{GAFF}^{[18]}$ for the ligand. The system was solvated with 35378 water molecules in a dodecahedral box and neutralized with two $\mathrm{Na}^{+}$ions (see Supporting Information). A $300 \mathrm{~ns}$ MD simulation of the dimer bound to two molecules of $\mathbf{1}$ was run to characterize the differences in the binding of the molecule to the two monomers and rationalize their different $K_{\mathrm{d}} \mathrm{s}$. The inter-conversion of the two monomer conformations was very fast (100 ns time scale). Fast hinge-bending motions in the 1-100 ns time scale have been reported previously, ${ }^{[10 a, c]}$ also in other kinases. ${ }^{[19]}$ Starting from a conformation where the monomer $\mathrm{A}$ is open $\left(\mathrm{A}^{*}\right)$ and the monomer $\mathrm{B}$ is closed (B), a transition to an opposite conformation $\mathrm{AB}^{*}$, where the second monomer is open, was observed after approximately $70 \mathrm{~ns}$. After another $100 \mathrm{~ns}$, the dimer went back to the original $\mathrm{A} * \mathrm{~B}$ conformation (Figure 4B). Initially, the two hydrogen bonds between 1 and the binding pocket of the open monomer A were less populated (48\% with Gln207 and 58\% with Ile209) than those of the closed monomer B $(75 \%$ and $83 \%$, respectively). The original binding pocket of monomer A contained more water molecules $(150 \pm 1$ in a $5 \AA$ solvation shell around the ligand) compared to monomer B (98 \pm 1 ; Figure S6). Owing to the insertion of water between the pyridinium end and the hydrophobic residues Tyr333, Tyr354, Trp420, and Trp423, large fluctuations of the ligand are observed at the choline binding site when the pocket is in the open conformation. This explains the higher affinity of $\mathbf{1}$ to the closed conformation that is initially assumed by monomer B (Figure S6). To confirm the allosteric coupling of the two monomers, we performed a second set of simulations. We removed the ligand bound to the most favorable $\mathrm{A} * \mathrm{~B}$ conformation and let the system relax for more than $50 \mathrm{~ns}$. In the simulation, we observed a very fast shift towards the opposite $\mathrm{AB}^{*}$ conformation, thus supporting the tight allosteric coupling between the two active site pockets (Figure S7). To optimize the interactions between the ligand and the hydrophobic residues in the choline binding site, monomer A* rapidly assumes a closed conformation which leads to the opening of monomer B. Principal component analysis (PCA) revealed that the closure of L1/L9 triggers the sliding of helix $\alpha 2$ and $\beta 4 \beta 5$. These are engaged in inter-monomer protein-protein interactions and cause an opposite movement of the N-lobe of monomer A, leading to the opening of its cavity.

The tight coupling between the monomers through the $\alpha 2 \beta 4 \beta 5-\alpha 7-\alpha 9-L 9$ elements explain the origin of the negative/ positive cooperativity reported previously for $\mathrm{CHOK}$ from different species. ${ }^{[6]}$ More importantly, our simulations show that the broken symmetry observed in the crystal structure can be dynamically recovered on average because of the fast exchange of the closed and open states assumed by the monomers.

In conclusion, we have investigated the binding mechanism of a compound that induces a negative-cooperativity allosteric mechanism in $\mathrm{CHOK} \alpha 1$ with a break of the dimer symmetry. We determined by protein X-ray crystallography that not only is a large conformational change induced by compound $\mathbf{1}$, but also that the conformation assumed by the two bound monomers are dissimilar. The consequent asymmetry of the dimer could explain the negative cooperativity, as was first proposed by Levitzki et al. ${ }^{[13]}$ and recently observed in the EGF receptor ${ }^{[20]}$ and human hemoglobin. ${ }^{[21]}$ However, MD simulations showed that the two monomers, coupled through the $\alpha 2 \beta 4 \beta 5-\alpha 7-\alpha 9$-L9 motifs, alternatively assume an open/semi-open or closed state with a fast interconversion rate, recovering on average the symmetry proposed in the original MWC model. Taken together, our data explain the molecular mechanism of the negative cooperativity, the inter-monomer coupling, and sets the foundation to design better and more selective inhibitors.

Received: December 3, 2012

Revised: January 16, 2013

Published online: February 25, 2013

Keywords: allosteric mechanisms . Choline kinase $\alpha 1$. elastic network analysis - molecular dynamics $\cdot$ X-ray diffraction 
[1] K. Glunde, Z. M. Bhujwalla, S. M. Ronen, Nat. Rev. Cancer 2011, $11,835-848$.

[2] A. Yalcin, B. Clem, S. Makoni, A. Clem, K. Nelson, J. Thornburg, D. Siow, A. N. Lane, S. E. Brock, U. Goswami, J. W. Eaton, S. Telang, J. Chesney, Oncogene 2010, 29, 139-149.

[3] a) B. S. Hong, A. Allali-Hassani, W. Tempel, P. J. Finerty, Jr., F. Mackenzie, S. Dimov, M. Vedadi, H. W. Park, J. Biol. Chem. 2010, 285, 16330-16340; b) F. W. Schueler, J. Pharmacol. Exp. Ther. 1955, 115, 127-143; c) J. G. Cannon, Med. Res. Rev. 1994, $14,505-531$.

[4] a) J. M. Campos, R. M. Sanchez-Martin, A. Conejo-Garcia, A. Entrena, M. A. Gallo, A. Espinosa, Curr. Med. Chem. 2006, 13, 1231-1248; b) D. Gallego-Ortega, T. Gomez del Pulgar, F. Valdes-Mora, A. Cebrian, J. C. Lacal, Adv. Enzyme Regul. 2011, 51, 183-194; c) D. Gallego-Ortega, A. Ramirez de Molina, M. A. Ramos, F. Valdes-Mora, M. G. Barderas, J. Sarmentero-Estrada, J. C. Lacal, PLoS One 2009, 4, e7819.

[5] a) A. Rodríguez-González, A. Ramirez de Molina, F. Fernandez, M. A. Ramos, M. del Carmen Nunez, J. Campos, J. C. Lacal, Oncogene 2003, 22, 8803-8812; b) A. Rodriguez-Gonzalez, A. Ramirez de Molina, J. Benitez-Rajal, J. C. Lacal, Prog. Cell Cycle Res. 2003, 5, 191-201; c) N. M. Al-Saffar, H. Troy, A. Ramirez de Molina, L. E. Jackson, B. Madhu, J. R. Griffiths, M. O. Leach, P. Workman, J. C. Lacal, I. R. Judson, Y. L. Chung, Cancer Res. 2006, 66, 427-434; d) A. Rodríguez-González, A. Ramirez de Molina, F. Fernandez, J. C. Lacal, Oncogene 2004, $23,8247-8259$.

[6] a) M. A. Brostrom, E. T. Browning, J. Biol. Chem. 1973, 248, 2364-2371; b) K. H. Kim, D. R. Voelker, M. T. Flocco, G. M. Carman, J. Biol. Chem. 1998, 273, 6844-6852.

[7] B. Rubio-Ruíz, A. Conejo-Garcia, P. Rios-Marco, M. P. Carrasco-Jimenez, J. Segovia, C. Marco, M. A. Gallo, A. Espinosa, A. Entrena, Eur. J. Med. Chem. 2012, 50, 154-162.

[8] J. P. Changeux, Annu. Rev. Biophys. 2012, 41, 103-133.
[9] D. E. Koshland, Jr., G. Nemethy, D. Filmer, Biochemistry 1966, $5,365-385$.

[10] a) B. L. de Groot, G. Vriend, H. J. Berendsen, J. Mol. Biol. 1999, 286, 1241 -1249; b) J. Ma, P. B. Sigler, Z. Xu, M. Karplus, J. Mol. Biol. 2000, 302, 303-313; c) R. Baron, N. A. Vellore, Proc. Natl. Acad. Sci. USA 2012, 109, 12509-12514.

[11] F. Bai, R. W. Branch, D. V. Nicolau, Jr., T. Pilizota, B. C. Steel, P. K. Maini, R. M. Berry, Science 2010, 327, 685-689.

[12] E. Malito, N. Sekulic, W. C. Too, M. Konrad, A. Lavie, J. Mol. Biol. 2006, 364, 136-151.

[13] A. Levitzki, W. B. Stallcup, D. E. Koshland, Jr., Biochemistry 1971, 10, 3371-3378.

[14] I. A. Balabin, W. Yang, D. N. Beratan, Proc. Natl. Acad. Sci. USA 2009, 106, 14253-14258.

[15] a) G. Saladino, F. L. Gervasio, Curr. Top. Med. Chem. 2012, 12, 1889-1895; b) P. Maragakis, M. Karplus, J. Mol. Biol. 2005, 352, $807-822$; c) P. C. Whitford, S. Gosavi, J. N. Onuchic, J. Biol. Chem. 2008, 283, 2042-2048.

[16] B. Hess, C. Kutzner, D. van der Spoel, E. Lindahl, J. Chem. Theory Comput. 2008, 4, 435-447.

[17] a) K. Lindorff-Larsen, S. Piana, K. Palmo, P. Maragakis, J. L. Klepeis, R. O. Dror, D. E. Shaw, Proteins Struct. Funct. Genet. 2010, 78, 1950-1958; b) R. B. Best, G. Hummer, J. Phys. Chem. B 2009, 113, 9004-9015.

[18] J. Wang, R. M. Wolf, J. W. Caldwell, P. A. Kollman, D. A. Case, J. Comput. Chem. 2004, 25, 1157-1174.

[19] a) Z. Palmai, L. Chaloin, C. Lionne, J. Fidy, D. Perahia, E. Balog, Proteins Struct. Funct. Genet. 2009, 77, 319-329; b) N. Smolin, R. Biehl, G. R. Kneller, D. Richter, J. C. Smith, Biophys. J. 2012, 102, 1108-1117.

[20] D. Alvarado, D. E. Klein, M. A. Lemmon, Cell 2010, 142, $568-$ 579.

[21] G. K. Ackers, J. M. Holt, J. Biol. Chem. 2006, 281, 11441-11443. 